\title{
Photoacoustic Spectroscopy by the Rear-Surface Detection for an Aluminum Foil Thin-Layer Chromatography Plate
}

\author{
Keiko OhSawa*1, Katsumi UchiYama*1, Yoshihiro Yoshimura*2, Asayo TaKasUka ${ }^{* 3}$, Kunio Mibe*4, \\ Kohei TAMURA*5, Yoshiki OHTANI*5 and Kazuo IMAEDA*2 \\ ${ }^{* 1}$ Department of Instrumental Analysis, Institute of Medical Chemistry, Hoshi University, \\ Ebara, Tokyo 142, Japan \\ *2 Department of Analytical Chemistry, Faculty of Pharmaceutical Science, Hoshi University, \\ Ebara, Tokyo 142, Japan \\ *3 Ushioda General Hospital, Shitanoya, Tsurumi, Yokohama 230, Japan \\ *4 Tokyo Hospital, Finance Ministry Printing Bureau, Nisigahara, Kita, Tokyo 114, Japan \\ *5 Ohkura National Hospital, Ohkura, Setagaya, Tokyo 157, Japan
}

\begin{abstract}
Photoacoustic spectroscopy by the rear-surface detection method was proposed for an aluminum-foil thin-layer chromatography (TLC) plate. The aluminum-foil TLC plate was attached to a photoacoustic cell (15 mm i.d.; chamber volume, $c a .0 .7 \mathrm{ml})$. The surface of the TLC plate was illuminated by a chopped laser-beam ( $30 \mathrm{~Hz}$; beam size $0.75 \mathrm{~mm} \phi$ ). The photoacoustic signal of the aluminum-foil TLC plate was detected by an electret condenser microphone located at the other end of the cell. The photoacoustic signal of the sample on the TLC plate was seen as a signal superposed on a background signal of the aluminum foil. Background signals caused by light absorption at the surface of aluminum foils were measured for foils $17,40,110 \mu \mathrm{m}$ thick. A $40 \mu \mathrm{m}$-thick aluminum foil showed the largest signal intensity. The amount of cobalt-PAN (1-(2-pyridylazo)-2-naphthol) chelate on a TLC plate was determined by the proposed method. A linear relationship between the amount of cobalt-PAN and the signal intensity was obtained within the range 20-300 ng. The detection limit of cobalt-PAN was about $5 \mathrm{ng}$. The reproducibility of measurements for $150 \mathrm{ng}$ cobalt-PAN on seven different TLC plates was 0.246 (RSD).
\end{abstract}

Keywords Photoacoustic, aluminum foil, thin-layer chromatography, rear surface detection, cobalt

Photoacoustic spectroscopy has been used as a highly sensitive tool for the characterization of solid materials without either destruction or tiresome pretreatment of samples. Many types of equipment have been proposed for measuring photoacoustic signals. These have mainly been classified into two types: one is based on the detection of the variation of pressure in a photoacoustic cell by use of a microphone ${ }^{1}$, and the other is based on the detection of elastic waves in a solid material by the use of a piezo-electric transducer. ${ }^{2}$ Both types have their merits and demerits based on the characteristics of the detector used. The former is most commonly used in photoacoustic detection due to both ease of construction and sensitivity. However, the gas microphone type requires an air-tight photoacoustic cell: that is to say, incident light must irradiate a sample through the cell window. This makes measurements troublesome and limits the application range.

We have previously reported a photoacoustic densitometer coupled with thin-layer chromatography (TLC). ${ }^{3-5}$ When a sample was placed on an aluminum-foil TLC plate $e^{6-8}$, the photoacoustic signal amplitude of the sample was smaller than that on a conventional glass- backed silica gel TLC plate. This resulted from both the large thermal diffusivity of the aluminum foil and a thin stationary phase (about $15 \mu \mathrm{m}$ ). However, aluminum-foil TLC plates occasionally showed 10 - or 50 times larger signals near the edge of the TLC plate than in the center. This phenomenon is considered to be due to synchronous vibration of the TLC plate caused by the incident light. The signal, however, was not reproducible.

We have found that when an aluminum-foil TLC plate (attached in the place of the window of the photoacoustic cell) was illuminated by chopped light, the foil transmitted photoacoustic signal through the cell chamber to a microphone. In order to obtain a reproducible photoacoustic signal of the aluminum-foil TLC plate, a photoacoustic apparatus was devised, which also allowed the photoacoustic measurement cellwindow to be free.

\section{Experimental}

Materials and methods

$T L C$ plate. Aluminum-foil TLC plates used were made 


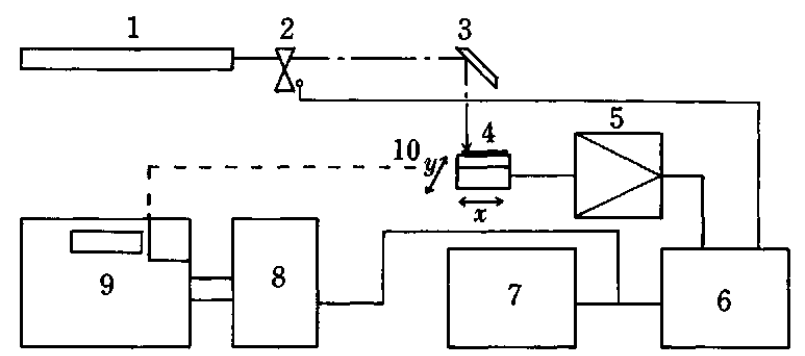

Fig. 1 Block diagram of the apparatus. 1, He-Ne laser; 2, light chopper $(30 \mathrm{~Hz}) ; 3$, mirror; 4, photoacoustic cell; 5, preamplifier; 6, lock-in amplifier; 7, recorder; 8, A/D converter; 9, microcomputer (Epson HC-20); 10, x,y table.

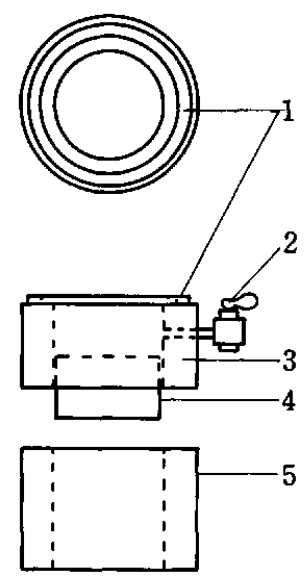

Fig. 2 Schematic diagram of the photoacoustic cell. 1, copper ring; 2, three-way cock for pressure release; 3 , cell body; 4 , electret condenser microphone; 5 , cell holder.

according to a method by Imaeda et al. ${ }^{6-8}$ The aluminum foil $(40 \mu \mathrm{m}$ thick) was cut into $5 \times 10 \mathrm{~cm}$ square pieces and treated with $2 \mathrm{M} \mathrm{NaOH}$ for $3 \mathrm{~min}$ to form an aluminum hydroxide gel on the surface. The plates were activated at $120^{\circ} \mathrm{C}$ for $3 \mathrm{~h}$, thus generating a stationary phase, with a thickness of about $15 \mu \mathrm{m}$. The aluminum-backed silica-gel thin-layer plates used were Merck type 5549 handy pack TLC plates.

Photoacoustic system. Figure 1 shows a block diagram of the apparatus. Details of the system have been described elsewhere. ${ }^{9}$ A photoacoustic cell made of copper is shown in Fig. 2. An aluminum-foil TLC plate was attached (using a vinyl acetate sticking agent) to a copper ring $(22.5 \mathrm{~mm}$ in internal diameter, $1.6 \mathrm{~mm}$ in thickness) which was then stuck to the cell body $(9.75 \mathrm{~mm}$ in internal diameter). In order to avoid any immediate change in the cell pressure, a three-way valve was facilitated. A chopped laser-beam was illuminated on the surface of the aluminum-foil TLC plate. Photoacoustic signals were detected by an electret condenser microphone located at the other end of the cell body. The chamber volume of the photoacoustic cell was about $0.7 \mathrm{ml}$.

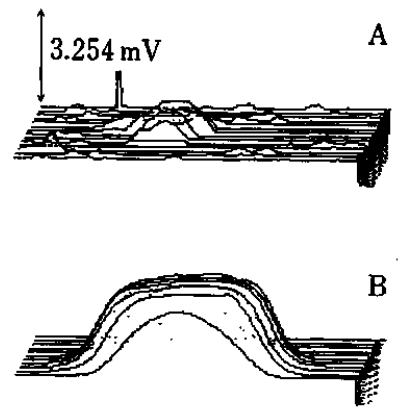

$\mathrm{C}$

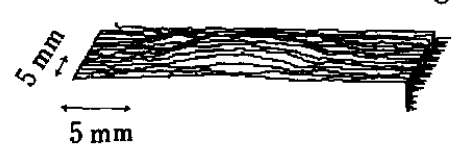

Fig. 3 Photoacoustic images of aluminum foil of various thickness obtained by $x, y$ scanning of the laser-beam. A: $17 \mu \mathrm{m}$; B: $40 \mu \mathrm{m}$; C: $110 \mu \mathrm{m}$.

Chemicals. All chemicals used were reagent grade. Cobalt was extracted into chloroform as 1-(2-pyridylazo)-2-naphthol (PAN) chelate according to a method by Galik. ${ }^{10}$

\section{Results and Discussion}

When aluminum foil was stuck to the cell body, the vibration character of the foil was determined by both the mass of the membrane as well as the compliance, as characterized by the tension and the Yang ratio of the material used. The basic frequency $\left(f_{01}\right)$ is summarized as,

$$
f_{01}=1 /(2 \pi \sqrt{M K})
$$

where $M$ is the mass of the membrane and $K$ is the compliance. Below $f_{01}$, the membrane was thought to vibrate uniformly. The absorbance of the aluminum foil at $632.7 \mathrm{~nm}$ (corresponding to the emission wavelength of the $\mathrm{He}-\mathrm{Ne}$ laser) was roughly estimated to be more than 0.01 . Untreated aluminum foil may generate heat by the illumination of a laser beam, resulting in the production of a background photoacoustic signal. In order to compare the background photoacoustic signals of aluminum foils of various thickness, 17, 40 and $110 \mu \mathrm{m}$ thick aluminum-foil pieces were attached to the photoacoustic cell. A laser beam (beam size, ca. $0.75 \mathrm{~mm} \phi$ ) was illuminated on the surface of these aluminum foils. The beam was $x, y$ scanned by two slide tables. Figure 3 shows plots of the photoacoustic amplitudes of those pieces of aluminum foil against the beam position on thin-layer plates. A $17 \mu \mathrm{m}$ aluminum foil thickness showed irregular signals and negative peaks, which is not shown in the figure due to the 
characteristics of the A/D converter used. This was, perhaps, due to some disturbance caused by surrounding noise as well as the low compliance of the aluminum foil. An aluminum-foil thickness of $40 \mu \mathrm{m}$ generated a uniform photoacoustic signal at the center of the membrane. Though the $110 \mu \mathrm{m}$ aluminum-foil thickness shows a uniform photoacoustic signal, the signal amplitude was very small. In this case, the membrane was so rigid that the photoacoustic signals generated at the illuminated surface of the aluminum foil were not transmitted through the cell chamber. When carbon black was spotted on these aluminum-foil pieces, the signals of the carbon black were superimposed on the background signals. This signal, minus the background signal, seemed to be proportional to the background signal. Of the three aluminum membranes, a thickness of $40 \mu \mathrm{m}$ resulted in the largest signal, suggesting that the photoacoustic signal of aluminum foil is mainly due to vibration of foil.

The photoacoustic signal of aluminum foil is thought to be affected by many parameters, such as the mass of the TLC plate and the thickness of the stationary phase. Aluminum-backed silica-gel TLC plates are most commonly used and have the following dimensions: an aluminum thickness of $110 \mu \mathrm{m}$, a silica-gel layer, and a thickness of $200 \mu \mathrm{m}$. The plate was attached to a photoacoustic cell and then carbon black was spotted ( $1 \mathrm{~mm} \phi)$ on the plate. Since the carbon black almost completely absorbed incident light at the surface, heat was generated near the surface of the stationary phase. Figure 4 shows plots of the photoacoustic signal $v s$. the laser-beam position on the plate. The signal phase for carbon black differed by 90 degrees, compared with that for the stationary phase. Negative signal images were obtained in between Figs. 4(B) and (C). Since heat

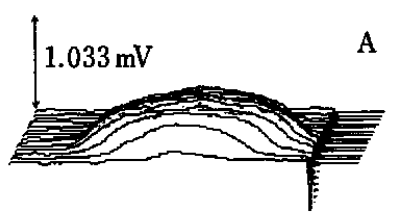

A
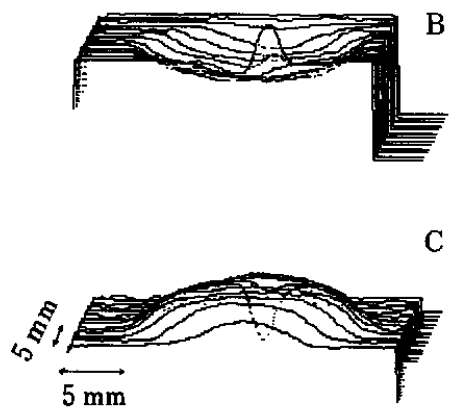

Fig. 4 Photoacoustic images of aluminum-backed silica-gel TLC plate obtained by $x, y$ scanning of the laser-beam. A: intact plate; B,C: carbon black was spotted on the TLC plate. B: phase was set on carbon black; C: phase was set on intact plate. is generated at the surface of the stationary phase, a certain delay exists between heat generation and the appearance of a photoacoustic signal. This suggests that the stationary phase must be sufficiently thin to transfer the signal to the aluminum membrane.

In order to confirm the relationship between the photoacoustic signal amplitude of $40 \mu \mathrm{m}$ thick aluminum-foil TLC plates and the mass of samples, various amounts of cobalt-PAN chelate extracted in chloroform were successively spotted on an aluminum foil TLC plate. Figure 5 shows the photoacoustic signal amplitudes for various amounts of the cobalt-PAN chelate. The phase of the signal in each spot was almost the same, regardless of the amount of cobalt used. Since background signals differ in each densitogram, signal intensities based on the light absorption of samples might vary in these densitograms. In order to correct sample signals, photoacoustic signals were divided by background signals, assuming that sample signals are generated in proportion to the background. Figure 6 shows the relationship between signal magnitudes and the amount of cobalt-PAN chelates by using the correction method mentioned above. A linear relation-

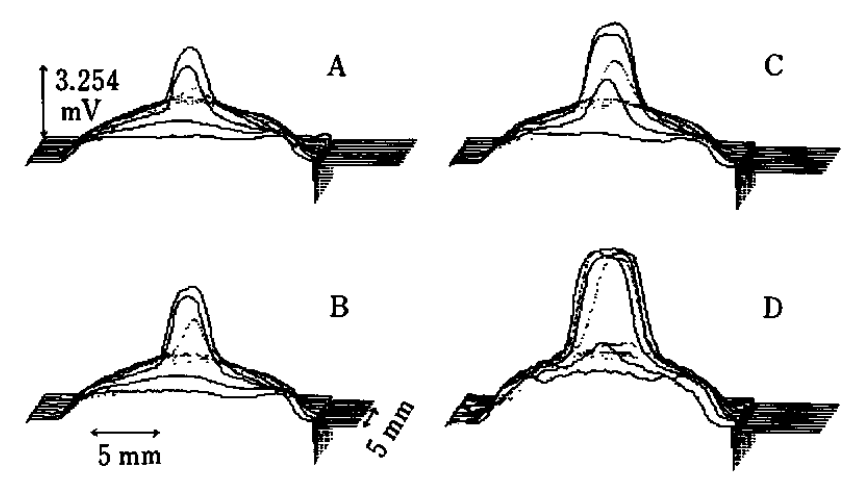

Fig. 5 Photoacoustic images for various amounts of cobaltPAN chelate on aluminum foil TLC plate obtained by $x, y$ scanning of the laser-beam. A: $40 \mathrm{ng} ; \mathrm{B}: 60 \mathrm{ng}$; C: $100 \mathrm{ng}$ : D: $200 \mathrm{ng}$ as the amount of cobalt.

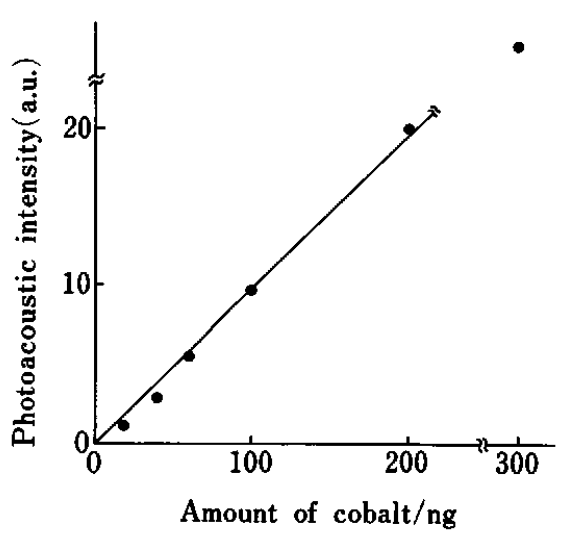

Fig. 6 Relationship between the amount of cobalt and the relative photoacoustic intensity. 
ship was obtained between 20 and $300 \mathrm{ng}$. The detection limit of the cobalt-PAN chelate on an aluminum-foil TLC plate was about $5 \mathrm{ng}(S / N=2)$. The reproducibility of photoacoustic signals corrected by the background signal for $150 \mathrm{ng}$ of the cobalt-PAN chelate on 7 different aluminum foil TLC plates was 0.137 (RSD); however, the value was 0.246 when the signal was not corrected in terms of the background intensity. The large RSD was thought to be due to the method of fixing the TLC plates to cells and to a heterogeneity of the stationary phase of the aluminumfoil TLC plate. Improvements of the technique to fix an aluminum foil TLC plate to a cell, the preparation technique of an aluminum foil TLC plate, and signal correction will make measurement, both more precise and reproducible.

The proposed method has potential for measuring invisible samples against incident light since the signal magnitude and phase are both parameters of the mass of samples on a membrane. In this case, however, the thickness of an aluminum-foil TLC plate should be reproducible. However, mechanisms regarding how a laser beam generates a photoacoustic signal in the cell chamber is not clear. These applications and theoretical analyses are presently being studied in our laboratory.

\section{References}

1. A. Rosencwaig and A. Gersho, J. Appl. Phys., 47, 64 (1976).

2. A. Hordrik and H. Schlossberg, Appl. Opt., 16, 101 (1977).

3. K. Imaeda, K. Ohsawa and K. Uchiyama, Bunseki Kagaku, 33, 320 (1984).

4. K. Imaeda, K. Ohsawa and K. Uchiyama, Bunseki Kagaku, 34, 346 (1985).

5. K. Imaeda, K. Ohsawa, K. Uchiyama, S. Nakamura and T. Tokieda, Anal. Sci., 2, 9 (1986).

6. K. Imaeda, K. Ohsawa and H. Shiraishi, Bunseki Kagaku, 27, 510, (1978).

7. K. Imaeda, K. Ohsawa and H. Shiraishi, Bunseki Kagaku, 28, 217 (1979).

8. K. Imaeda, K. Ohsawa and H. Shiraishi, Bunseki Kagaku, 29, 53 (1980).

9. K. Ohsawa, K. Uchiyama, S. Suzumura, K. Imaeda and K. Tamura, Bunseki Kagaku, 37, 147 (1988).

10. A. Galik and A. Vincourova, Anal. Chim. Acta, 46, 113 (1969).

(Received February 23, 1990) (Accepted June 28, 1990) 